transmissible and failure to eradicate it from the cervix will expose the male partner to risk. It may be that in time increases in penile cancer will also become evident-with the wart virus as the prime suspect.

Birmingham Maternity Hospital, Queen Elizabeth Medical Centre, Edgbaston, Birmingham B15 2TG

Chief Medical Officer. Sexually transmitted diseases extract from the annual report of the Chief Medical Officer of the Department of Health and Social 206-10.

SIR,-Most genital and extragenital warts undergo spontaneous resolution (10 March, p 735). Cervical human papillomavirus infection is usually discovered only in patients with cytological abnormality referred for colposcopic assessment. As most of these patients are subsequently treated for an associated dysplastic lesion, the natural progression of human papillomavirus infection has not been studied. A recent study in the Birmingham and Midland Hospital for Women showed that of 48 women referred for evaluation of abnormal cytology with cervical human papillomavirus infection without associated cervical intraepithelial neoplasia 27 $(56 \%)$ showed no cytological or colposcopic abnormalities after a median follow up of 10 months. This suggests that cervical human papillomavirus infection is often self limiting and with resolution of the viral infection cytological, colposcopic, and histological abnormalities may regress.

Alternatively, incorporation of the viral genome into metaplastic cells may produce an irreversible progression of dysplastic change making it imperative to treat these lesions whenever found. Dr Albert Singer and others cite the excellent results achieved with local destructive techniques in the treatment of cervical intraepithelial neoplasia. This is based on the premise that all histologically abnormal epithelium can be identified by colposcopic assessment, but this assumption may be invalid in human papillomavirus infection of the cervix. Colposcopically normal mature squamous epithelium may harbour sufficient quantities of the viral genome to maintain a productive infection after destruction of all colposcopically atypical epithelium. In addition the human papillomavirus genome may be sequestered in tissue below the depth of destruction normally achieved by local destructive treatment. A controlled study of the use of local destructive techniques in the management of cervical human papillomavirus infection is needed.

Birmingham and Midland Hospital for Women,
Birmingham B11 $4 \mathrm{HL}$

\section{ABC of sexually transmitted diseases}

SIR,-Dr E M C Dunlop (25 February, p 641) worries too much about the aptness of the term "genitourinary medicine" to describe our specialty. I agree with him that it is not perfect but it is immeasurably better than the titles venereology or venereological medicine. To the man in the street venereology or venereal disease means something to do with syphilis or gonorrhoea. As these illnesses now account for less than $16 \%$ of the patients seen in the clinics, it seems unkind to add to patients' fears no matter how illogical this may seem to doctors.

In 1970 in response to a letter about the difficulty in recruitment to venereology, I wrote, "To my mind there is no simple, single measure which could do so much for the status of the specialty and for the well-being of the majority of our patients as a change of name, such as that suggested in your leading article of 21 February, p 447."' The success of the term genitourinary medicine may be measured by the fact that today three quarters of clinics have adopted this title.

Finally, I agree with him that one of our major tasks is to control sexually transmitted disease, and I think the figures show that compared with many other countries we do a most effective job. Reviewing the past five working days during which I conducted 10 clinic outpatient sessions in two separate hospitals, only $40 \%$ of my work was connected with venereal disease and sexually transmitted diseases. The problems in any clinic will depend to some extent on the population it serves and the interests of the doctors working there, but I do not think my experience is particularly unusual.

Department of Genitourinary
Medicine,
Westminster Hospital,
London SW1P 2AP
'Oates JK. Recruits for venereology. Br Med $\mathcal{F} 1970$;
ii:296.
ii:296.

\section{"Third drug" trial}

SIR,-A major part of the analysis by Dr D McAreavey and others (14 January, p 106) relied on the comparison of reductions in systolic blood pressure for the patients who successfully completed treatment with a third drug. The reduction in blood pressure was defined as the difference between the blood pressure at the end of a run in period (which had to be greater than $140 / 95 \mathrm{~mm} \mathrm{Hg}$ ) and that at the end of the third drug phase. A target of less than $140 / 95 \mathrm{~mm} \mathrm{Hg}$ was set, and dosage schedules were manipulated to try to achieve this.

Although it is not as certain as Dr McAreavey and colleagues suggest, even fixed dose antihypertensive treatment may achieve greater reductions in blood pressure for those with initially higher pressures. The authors seem unaware, however, of two other reasons why the reduction achieved is likely to be greatest for those with initially highest pressures: the attempt to achieve target pressures and the effect of regression to the mean. The effect of regression to the mean has been discussed often, ${ }^{1}$ notably in relation to blood pressure. ${ }^{2}$ The authors' analysis entailed regressing systolic blood pressure reduction on initial systolic blood pressure, but a positive correlation is to be expected between reduction in blood pressure and initial value even for ineffective treatment, which is why such analyses are strongly discouraged. ${ }^{3}$ Furthermore, their figure showed only the fitted regression lines and not the widely ranging data values, and the fact that the low average initial systolic blood pressure of those successfully completing the minoxidil regimen was off the edge of their figure was obscured. The average reduction in lying blood pressure for the minoxidil group was similar or even less than that for the other four active regimens studied; this is not evident from their figure. A large proportion of subjects were unable to complete their regimens (through side effects and so on), ranging from $30 \%$ to $78 \%$ for the different active drug groups. An analysis restricted to those who completed the study should lead to only tentative conclusions.
The authors' assertion that minoxidil was more potent than all other agents is not justified.

The statistical methods used in other parts of the paper were not identified. In particular, it is not clear whether appropriate allowance was made in the quoted significance levels for the multiple comparison of pairs out of the five active drugs. It is well known that the naive multiple testing of pairs of differences between a number of groups gives exaggerated significance levels. Testing each pair of active drugs at a nominal $5 \%$ significance level yields a false positive rate of $40 \%$.

A test of efficacy of such drug regimens should be based on all the patients randomly allocated to each regimen, and not just on the patients successfully completing the course. Because so many patients were withdrawn, it seems inappropriate to use the reduction in blood pressure as a criterion, and we would recommend the comparison of the proportion of patients responding-that is, not being withdrawn, and attaining targets of blood pressure reduction-among the five active drug groups. This analysis is not affected by regression to the mean, and the appropriate $\chi^{2}$ test based on the authors' figures yields $\chi^{2}-3.73, \mathrm{p}=0.44$ Thus there is no evidence to reject the hypothesis of equal efficacy of the five active drugs. This analysis does not directly weight the severity of side effects, which of course have to be discussed explicitly.

The strongly asserted conclusions given by Dr McAreavey and colleagues are thus unsupported when the results are examined closely.

Douglas G Altman

Division of Computing an

Statistics,

Clinical Research Centre,

Harrow,

Mece

SIMON G THOMPSON

MRC Epidemiology and

Medical Care Unit,
Northwick Park Hospital,

Northwick

Harrow,

Oldham PD. A note on the analysis of repeated measurements on the same subjects. $\mathcal{F}$ Chronic Dis

Shepard DS, Finison LJ. Blood pressure reductions correcting for regression to the mean. Prev Med 1983;12:304-17.

- Altman DG, Gore SM, Gardner MJ, Pocock SJ. Statistical guidelines for contributors to medical

***The authors reply below.-ED, BMf.

Sir,-Mr D G Altman and Mr S G Thompson criticise, firstly, the use of regression analysis to compare different drug regimens and the figure illustrating this.

Having fitted, separately for each regimen, a regression of reduction in blood pressure on initial blood pressure, we compared expected reductions for a given initial pressure. For instance, for an initial lying systolic blood pressure of $180 \mathrm{~mm} \mathrm{Hg}$ minoxidil was significantly more hypotensive than any of the other regimens. For an initial pressure of $160 \mathrm{~mm} \mathrm{Hg}$ minoxidil was significantly more hypotensive than prazosin, hydralazine, or placebo. Initial pressure is a classic concomitant variable ${ }^{\mathbf{1}}$; the fact that it is correlated with reduction in pressure, whether the regimen is effective or not, is the very reason for including initial pressure in the analysis. The precise explanation of the correlation between reduction and initial pressure is irrelevant to the statistical comparison of regimens, provided that initial pressure is not itself affected by that regimen. The inference by $\mathrm{Mr}$ Altman and $\mathrm{Mr}$ Thompson that we are unaware of reasons for the correlation is incorrect. In our trial, initial pressure was measured before a patient started taking the third drug. Mr Altman and $\mathrm{Mr}$ Thompson appear to favour a comparison of average reductions ignoring initial values. This is obviously more sensitive than merely comparing average final pressures, and the reason for the 